\title{
Superoperator Analysis of Entanglement in a Four-Qubit Cluster State
}

\author{
Yaakov S. Weinstein, ${ }^{1}$ Jay Feldman,, , ${ }^{1}$ Jacob Robins, ${ }^{1, \oplus}$ Jason Zukus, ${ }^{1,}$ 团 and Gerald Gilbert ${ }^{1}$ \\ ${ }^{1}$ Quantum Information Science Group, MitRe, 260 Industrial Way West, Eatontown, NJ 07224, USA
}

\begin{abstract}
In this paper we utilize superoperator formalism to explore the entanglement evolution of fourqubit cluster states in a number of decohering environments. A four-qubit cluster state is a resource for the performance of an arbitrary single logical qubit rotation via measurement based cluster state quantum computation. We are specifically interested in the relationship between entanglement evolution and the fidelity with which the arbitrary single logical qubit rotation can be implemented in the presence of decoherence as this will have important experimental ramifications. We also note the exhibition of entanglement sudden death (ESD) and ask how severely its onset affects the utilization of the cluster state as a means of implementing an arbitrary single logical qubit rotation.
\end{abstract}

PACS numbers: 03.67.Mn, 03.67.Bg, 03.67.Pp

\section{INTRODUCTION}

Entanglement is a uniquely quantum mechanical phenomenon in which quantum systems exhibit correlations above and beyond what is classically possible. Entangled systems are thus an important resource for many quantum information processing protocols including quantum computation, quantum metrology, and quantum communication [1]. In the area of quantum computation, certain entangled states play a unique role as the basic resource for measurement-based quantum computation. The cluster state in particular allows for quantum computation to proceed via single qubit measurements after creation of the cluster state [2].

An important area of research is to understand the possible degradation of entanglement due to decoherence. Decoherence, stemming from unwanted interactions between the system and environment, is a major challenge confronting experimental implementations of quantum computation, metrology, and communication [3]. Decoherence may be especially detrimental to highly entangled states [4] and, indeed, much work has been done on studying the effects of decoherence on cluster states [5].

An extreme manifestation of the detrimental effects of decoherence on entangled states is "entanglement sudden death" (ESD), in which entanglement within a system is completely lost in a finite time [6, 7] despite the fact that the loss of system coherence is asymptotic. This aspect of entanglement has been well explored in the case of bi-partite systems and there are a number of studies looking at ESD in multi-partite systems [8 13] including the four qubit cluster state 14. In addition, there have been several initial experimental ESD studies 15].

In this paper we study the entanglement evolution of a four-qubit cluster state which can be used as the basic re-

*Present address: Johns Hopkins University, Baltimore, MD 21201, USA

${ }^{\dagger}$ Present address: University of Pennsylvania, Philadelphia, PA 19104, USA

${ }^{\ddagger}$ Present address: Princeton University, Princeton, NJ 08544, USA source to perform an arbitrary single (logical) qubit rotation via cluster state quantum computation. We analyze the effects of various decoherence models on the entanglement of the pre-measurement state and compare the entanglement behavior to the accuracy with which the decohered state can be used to implement the desired arbitrary single qubit rotation. To completely characterize the effects of decoherence we make use of superoperator representations and aspects of quantum process tomography. Quantum process tomography is an experimental protocol which is used to completely determine (open) system dynamics. The information gleaned from quantum process tomography can, in turn, be used to determine a wealth of accuracy measures. One would expect that the proper working of cluster state based quantum computation would be strongly dependent on the amount of entanglement present in the pre-measurement cluster state. Thus, an explicit analysis of the strength of this dependence, especially when attempting to perform basic computational gates, is essential for experimental implementations of cluster state quantum protocols.

A secondary aim of this paper is to analyze the effect of ESD on the implementation of the single logical qubit rotation. The ESD phenomenon is of interest on a fundamental level and important for the general study of entanglement. However, it is not yet clear what the effect of ESD is on quantum information protocols. Are different quantum protocols helped, hurt, or left intact by ESD? Previous results suggest a possible connection between the loss of certain types of entanglement in the four qubit cluster state and the fidelity with which measurements on the four qubit state will lead to the desired state on the remaining, unmeasured qubits 14]. The current paper expands these results by exploring additional decoherence mechanisms and calculating state independent accuracy measures such as the gate fidelity. Other explicit studies of the effect of ESD on quantum information protocols include the three-qubit phase flip code, a four qubit decoherence free subspace and a three qubit noiseless subsystem [13, 16]. None of these studies find a correlation between the accuracy of the protocol implementation and the advent of ESD. 


\section{CLUSTER STATES}

The cluster state [17] is a specific type of entangled state that can be used as a resource for measurementbased quantum computation [2]. A cluster state can be constructed by rotating all qubits into the state $|+\rangle=$ $\frac{1}{\sqrt{2}}(|0\rangle+|1\rangle)$ and applying control phase (CZ) gates, $\operatorname{diag}(1,1,1,-1)$, between desired pairs. In a graphical picture of a cluster state, qubits are represented by circles and pairs of qubits that have been connected via $\mathrm{CZ}$ gates are connected by a line. A cluster state with qubits arranged in a two-dimensional lattice, such that each (non-edge) qubit has been connected via $\mathrm{CZ}$ gates with its four nearest neighbors, suffices for universal QC.

After constructing the cluster state, any quantum computational algorithm can be implemented using only single-qubit measurements performed along axes in the $x-y$ plane of the qubit, i.e. the plane spanned by $|+\rangle=$ $\frac{1}{\sqrt{2}}(|0\rangle+|1\rangle),|+i\rangle=\frac{1}{\sqrt{2}}(|0\rangle+i|1\rangle)$. These processing measurements are performed by column, from left to right, until only the last column remains unmeasured. The last column contains the output state of the quantum algorithm which can be extracted by a final readout measurement. One can view each row of the clusterstate lattice as the evolution of a single logical qubit in time. Two (logical) qubit gates are performed via connections between two rows of the cluster state. CZ gates in particular are 'built-in' to the cluster state and simple measurement on two connected qubits in different rows automatically implements the gate.

Measurement of a physical qubit in the cluster state at an angle $\phi$ from the $x$-axis in the $x-y$ plane implements a rotation on the logical qubit given by $X(\pi m) H Z(\phi)$, where $H=\frac{1}{\sqrt{2}}\left(\begin{array}{cc}1 & 1 \\ 1 & -1\end{array}\right)$ is the Hadamard gate, and $Z(\alpha)(X(\alpha))$ is a $z$ - $(x-)$ rotation by an angle $\alpha[18$. The dependence of the logical operation on the outcome of the measurement is determined by the value of $m=0,1$ for measurement outcome $-1,+1$, respectively. An arbitrary single logical qubit rotation can be implemented via three such measuremnts yielding:

$$
H Z\left(\theta_{1}+\pi m_{\theta_{1}}\right) X\left(\theta_{2}+\pi m_{\theta_{2}}\right) Z\left(\theta_{3}+\pi m_{\theta_{3}}\right),
$$

where $\left(\theta_{1}, \theta_{2}, \theta_{3}\right)$ are the Euler angles of the rotation. As an example, by drawing the Euler angles according to the Haar measure, a random single-qubit rotation can be implemented.

We explore an arbitrary single (logical) qubit clusterbased rotation performed on an arbitrary initial state in a decohering environment. To construct the relevant cluster, a qubit is placed in the desired initial state $\left|\psi_{\text {in }}(\alpha, \beta)\right\rangle=\cos \alpha|0\rangle+e^{i \beta} \sin \alpha|1\rangle$, where $\rho_{\text {in }}(\alpha, \beta)=$ $\left|\psi_{\text {in }}(\alpha, \beta)\right\rangle\left\langle\psi_{\text {in }}(\alpha, \beta)\right|$. Three additional qubits (numbered 2-4) are rotated into the $|+\rangle$-state and CZ gates are then applied between the original qubit and 2,2 and 3 , and 3 and 4 . The four qubit initial (pure) state is thus $\left|\psi_{4 I}(\alpha, \beta)\right\rangle=C Z_{34} C Z_{23} C Z_{12}\left(\left|\psi_{\text {in }}(\alpha, \beta)\right\rangle \otimes|+\rangle^{\otimes 3}\right)$ or $\rho_{4 I}(\alpha, \beta)=\left|\psi_{4 I}(\alpha, \beta)\right\rangle\left\langle\psi_{4 I}(\alpha, \beta)\right|$.

\section{ENTANGLEMENT MEASURES}

To quantify and monitor entanglement in the above constructed types of cluster states as they undergo decoherence we use an entanglement measure known as the negativity, $N$, defined as the most negative eigenvalue of the parital transpose of the system density matrix [19]. There are a number of inequivalent forms of the negativity for any four qubit system: the partial transpose may be taken with respect to any single qubit, $N^{(j)}$, or the partial transpose may be taken with respect to any two qubits: $N^{(j, k)}$. The negativity thus defined does not differentiate different types of entanglement. Furthermore, due to the possible presence of bound entanglement, the disappearance of all measureable negativity does not guarantee that the state is separable. However, the presence of negativity does ensure the presence of distillable entanglement in the system.

A method of monitoring specifically four qubit cluster type entanglement is via the expectation value of the state with respect to an appropriate entanglement witness [20]. Entanglement witnesses are observables with positive or zero expectation value for all states not in a specified entanglement class and a negative expectation value for at least one state of the specified entanglement class. Entanglement witnesses may allow for an efficient, though imperfect, means of experimentally determining whether entanglement is present in a state (as opposed to inefficient state tomography). This is especially important for experiments with any more than a few qubits as it may be the only practical means of deciding whether or not sufficient entanglement is present in the system. The entanglement witnesses used here are specifically designed to detect four qubit cluster type entanglement of the kind exhibited by states of the form $\rho_{4 I}(\alpha, \beta)$. In Ref. 21] an entanglement witness is constructed for a cluster state in which the first qubit is $|+\rangle$. It is given by $\mathcal{W}_{+}=\mathbb{1} / 2-\rho_{4 I}(\pi / 4,0)$. For the current study we modify this witness by a phase rotation of angle $\beta$ on the first qubit yielding witnesses of the form:

$$
\mathcal{W}_{\beta}=\mathbb{1} / 2-e^{-i \beta \sigma_{z}^{1} / 2} \rho_{4 I}(\pi / 4,0) e^{i \beta \sigma_{z}^{1} / 2},
$$

where $\sigma_{z}^{k}$ is the Pauli $z$ spin operator on qubit $k$ and $\beta$ is the phase of the initial state $\left|\psi_{i n}(\alpha, \beta)\right\rangle$. This witness more accurately determines whether the cluster states of interest in this work have any four-qubit cluster entanglement.

\section{SUPEROPERATOR REPRESENTATION}

We would like to completely describe the evolution of the single logical qubit undergoing an arbitrary clusterbased rotation in the presence of decoherence. To do 
so we need to account for both the decoherence and the measurements of the (three) physical qubits. For this study we assume that there is no interaction between the qubits of the cluster state (beyond the initial conditional phase gates used to construct the cluster state). We further assume that all decoherence occurs after construction of the cluster state but before measurements. Measurement is done on each of the first three qubits in bases at angle $\theta_{i}, i=1,2,3$, from the positive $x$-axis. As noted above, the measurement bases are chosen so as to implement the desired logical qubit rotation. After measurement the final state of the logical qubit resides on the fourth, unmeasured, physical qubit and is a function of the initial state of the logical qubit $\left|\psi_{\text {in }}(\alpha, \beta)\right\rangle$, the decoherence strength, $p$, and the three measurement angles, $\theta_{i}: \rho_{\text {out }}=\rho_{\text {out }}\left(\alpha, \beta, p, \theta_{1}, \theta_{2}, \theta_{3}\right)$.

To construct the dynamical superoperator of the one qubit logical gate we follow the method described in [3, 22]. We construct the appropriate cluster, apply decohering evolution, and perform the desired measurements on a set of states, $\left|\psi_{4 I}(\alpha, \beta)\right\rangle$, which span the one qubit Hilbert space (Hilbert space dimension $N=2$ ). From this we can construct the $N^{2} \times N^{2}$ Liouvillian superoperator, $S$, where

$$
S\left(p, \theta_{1}, \theta_{2}, \theta_{3}\right) \rho_{\text {in }}(\alpha, \beta)=\rho_{\text {out }}\left(\alpha, \beta, p, \theta_{1}, \theta_{2}, \theta_{3}\right) .
$$

Note that in Liouvillian space, density matrices are column vectors of dimension $N^{2} \times 1$. From the superoperator $S$ we can construct the corresponding $N \times N$ Kraus operators following [23]. An analysis of the Kraus operator representation of the scenarios outlined below is done in the Appendix B.

\section{A. Accuracy Measures}

There are two accuracy measures that we find useful for our analysis and that we use to compare the accuracy of the implemented gate to the evolution of the entanglement. These measures quantify how well the system performed the desired operation and are thus vital in experimental work. The first accuracy measure we utilize is the cluster state fidelity of the four qubit state, $\rho_{4 F}(\alpha, \beta, p)$, before measurement but after decoherence as a function of $p$. This is given by:

$$
F^{c}=\operatorname{Tr}\left[\rho_{4 I}(\alpha, \beta) \rho_{4 F}(\alpha, \beta, p)^{\dagger}\right] .
$$

This is a simple measure which tells how close the actual final state is to the desired one in the presence of decoherence. The second accuracy measure is the gate fidelity of the attempted single (logical) qubit rotation, $U\left(\theta_{1}, \theta_{2}, \theta_{3}\right)$. The gate fidelity quantifies the accuracy with which the attempted evolution was achieved independent of the initial state of the system. The superoperator allows us to calculate the gate fidelity via:

$$
F^{g}=\operatorname{Tr}\left[S\left(0, \theta_{1}, \theta_{2}, \theta_{3}\right) S\left(p, \theta_{1}, \theta_{2}, \theta_{3}\right)^{\dagger}\right] .
$$

where

$$
S\left(0, \theta_{1}, \theta_{2}, \theta_{3}\right)=U\left(\theta_{1}, \theta_{2}, \theta_{3}\right) \otimes \operatorname{Conj}\left(\mathrm{U}\left(\theta_{1}, \theta_{2}, \theta_{3}\right)\right) .
$$

We next look at decohering environments of experimental interest: phase damping, amplitude damping and depolarization. In all three we explore the entanglement evolution as a function of decoherence strength assuming that the decoherence occurs prior to measurement. Measurements are performed on the decohered state and thus the evolution of the fidelity of the implemented operation can be compared to the evolution of the entanglement. Our goal is to see what correlations exist between entanglement degradation and the accuracy with which the cluster state can be used to implement the desired single logical qubit rotation. We will also note occurrences of ESD and what effect this phenomenon may have on the ability of the system to implement the desired rotation.

\section{DECOHERING ENVIRONMENTS}

In this paper we discuss the effects of three different decohering environments on the four qubit cluster state. They are: independent qubit phase damping, amplitude damping, and depolarization. Each of these environments is completely described by a Kraus operator representation. The Kraus operator representation for the phase damping environment is given by:

$$
K_{1}=\left(\begin{array}{cc}
1 & 0 \\
0 & \sqrt{1-p}
\end{array}\right), \quad K_{2}=\left(\begin{array}{cc}
0 & 0 \\
0 & \sqrt{p}
\end{array}\right),
$$

for the amplitude damping environment:

$$
K_{1}=\left(\begin{array}{cc}
1 & 0 \\
0 & \sqrt{1-p}
\end{array}\right), K_{2}=\left(\begin{array}{cc}
0 & \sqrt{p} \\
0 & 0
\end{array}\right),
$$

and for the depolarizing environment:

$$
K_{1}=\left(\begin{array}{cc}
\sqrt{1-\frac{3 p}{4}} & 0 \\
0 & \sqrt{1-\frac{3 p}{4}}
\end{array}\right), K_{\ell}=\frac{\sqrt{p}}{2} \sigma_{\ell},
$$

where the $\sigma_{\ell}$ are the Pauli spin operators, $\ell=x, y, z$. In each case we have defined a decoherence strength parameter $p$ whose exact behavior as a function of time is left unspecified so as to accomodate various possible experimentally relevant behaviors. As an example, one might have $p=1-e^{-\kappa \tau}$ where $\tau$ is time and $\kappa$ is the decay constant. In the case of independent qubit dephasing, this decoherence behavior would decay off-diagonal terms of the density matrix as a power of $e^{-\kappa \tau}$ and thus go to zero (i. e. $p \rightarrow 1$ ) only in the limit of infinite times. We also assume equal decoherence for all four qubits.

\section{RESULTS}

Starting with the general state $\rho_{4 I}(\alpha, \beta)$ we separately apply each of the decohering environments with arbitrary 
TABLE I: Summary of entanglement and fidelity results for the three explored decohering environments. The columns show values of $p$ at which ESD is exhibited, values of $p$ where the entanglement witness cannot detect entanglement, gate fidelity, and the state fidelity, where $q=p-1$ and $\tilde{p}=\sqrt{1-p}$.

\begin{tabular}{||c||c|c|c|c||}
\hline & $\operatorname{ESD}\left(N^{(j)}=0\right)$ & $\operatorname{Tr}\left[\rho \mathcal{W}_{\beta}\right]=0$ & $F^{g}$ & $F^{C}$ \\
\hline \hline Dephasing & $N^{(1)}, N^{(1,2)}: p=2(\sqrt{2}-1)$ & $p \lesssim .5$ & $\frac{1}{16}\left(10+6 \tilde{p}+p(p-6 \tilde{p}-7)+q(p+2 \tilde{p}-2) \cos 2 \theta_{2}\right.$ & $\frac{1}{32}(16(1+\tilde{p})+p(p-6 \tilde{p}-14)$ \\
& $N^{(1,3)}: p \simeq .938$ & & $\left.+2 q(p+2 \tilde{p}-2) \cos \theta_{2}^{2} \cos 2 \theta_{3}\right)$ & $-p(p-2 \tilde{p}-2) \cos 4 \alpha)$ \\
\hline Amplitude & none & $p<.2$ & same as dephasing & see figure \\
Damping & & & & \\
\hline Depolarizing & all $N^{(j)}: p \leq .45$ & $p \lesssim .2$ & $\frac{1}{8}\left((p-2)(-4+p(7+p(p-6)))+q^{2} p^{2} \cos 2 \theta_{2}\right)$ & $\frac{1}{32}(p-2)^{2}(3 p(3 p-5)+8-q p \cos 4 \alpha)$ \\
\hline
\end{tabular}

decoherence strength $p$ and determine the entanglement in the output state $\rho_{4 F}(\alpha, \beta, p)$. For the depolarizing environment the eigenvalues of the partially tranposed states can be determined analytically. For the other environments the eigenvalues for each $\alpha$ and $p$ must be determined numerically. The entanglement evolution is shown in Figs. 1-3 and summarized in Table [ We also calculate cluster state fidelity comparing the state before and after decoherence.

The entanglement of the system before or after decoherence is completely independent of $\beta$. beta arises as a rotation, $\exp \left[-i \frac{\beta}{2} \sigma_{z}\right]$, on the state $\left|\psi_{i n}(\alpha, 0)\right\rangle$. This rotation commutes with $\mathrm{CZ}$ gates and, as single qubit rotations do not effect the entanglement, the entanglement of the state $\left|\psi_{4 I}(\alpha, \beta)\right\rangle$ is independent of $\beta$. The $\sigma_{z}$ rotation which gives rise to $\beta$ also commutes with the Kraus operators and of the phase damping and amplitude damping environments. Thus, the entanglement of the cluster states under these types of decoherence is also independent of $\beta$. The depolarizing environment, however, includes (two) Kraus operators proportional to $\sigma_{x}$ and $\sigma_{y}$ which do not commute with the $\sigma_{z}$ rotation. Nevertheless, the rotation amounts to simply reorienting $\sigma_{x}$ and $\sigma_{y}$ and thus, taken together, the $\sigma_{z}$ rotation can be applied after the decoherence with no effect on the final state.

We then apply measurements to the first three qubits along an axis in the $x-y$ plane at angles $\theta_{1}, \theta_{2}, \theta_{3}$ from the x-zxis. From this we determine the superoperator, $S\left(p, \theta_{1}, \theta_{2}, \theta_{3}\right)$, of the attempted arbtrary rotation. These superoperators are explicitly shown in Appendix A. From $S\left(p, \theta_{1}, \theta_{2}, \theta_{3}\right)$ we can compute the state independent gate fidelity as a function of the attempted rotation and the decoherence strength and determine the output state for any input state. Explicit equations for

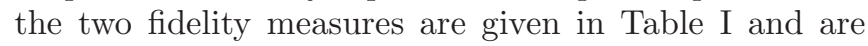
depicted in Figs. 4-6.

There are a number of differences in the entanglement evolution between the three decohering environments. Entanglement degrades most slowly in the dephasing environment before exhibiting ESD at high values of $p$. In the amplitude damping environment the entanglement degrades more quickly but never exhibits ESD, and in the depolarizing environment the entanglement degrades most quickly and ESD is exhibited for low values of $p$. In addition, different states lose entanglement at different rates depending on the decohering environement. For example, $N^{(1,2)}$ in all states degrades uniformly in the
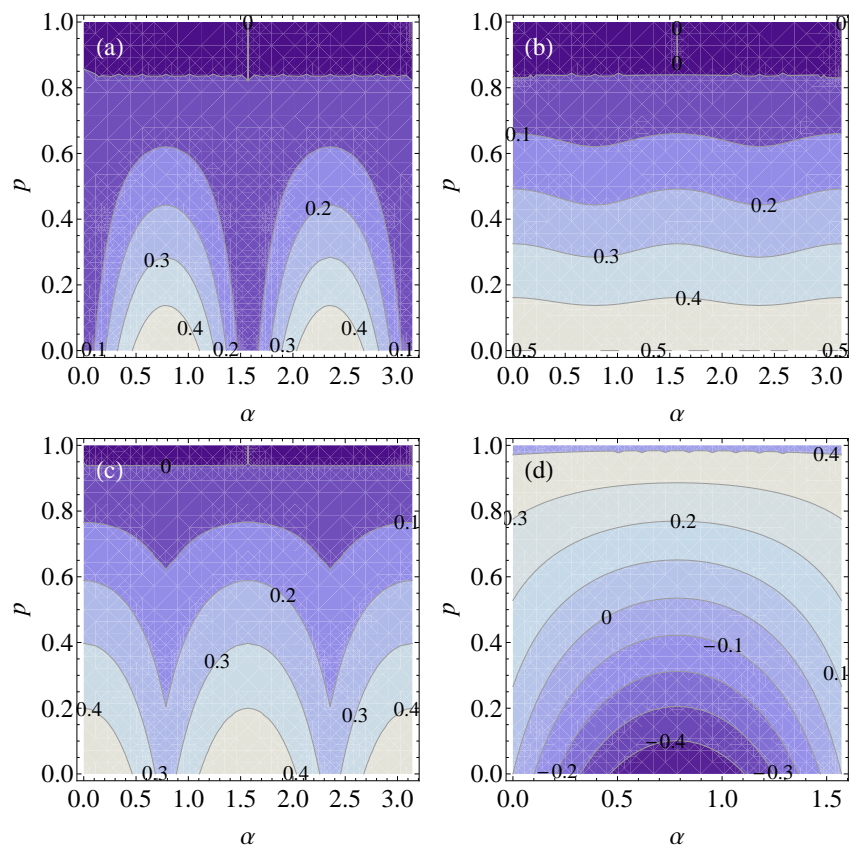

FIG. 1: (Color online) Negativity as a function of dephasing strength, $p$, and initial state parameterized by $\alpha$ : (a) $N^{(1)}$, partial transpose taken with respect to the first qubit, (b) $N^{(1,2)}$, partial transpose taken with respect to the first two qubits, (c) $N^{(1,3)}$ partial transpose taken with respect to qubits one and three. (d) Evolution of the expectation value of the entanglement witness as a function of initial state (the expectation value is not dependent on $\beta$ ) and decoherence strength. Notice that the dephasing strength at which the expectation value goes to zero is dependent on $\alpha$ and is well below the point where ESD is exhibited for $N^{(1)}$. The four qubit cluster entanglement can only be observed at low levels of decoherence, $p \lesssim .5$.

depolarizing environment but not in the other environements. This is most likely due to uniform effect of the depolarizing environment over the Bloch sphere.

With respect to detecting entanglement via the entanglement witness, we find for all decohering environments that the detection of four qubit cluster entanglement goes to zero much more quickly than any of the entanglement measures (and goes to zero for the amplitude damping environment though no ESD is exhibited). This shows a quick demise specifically for the four-qubit cluster entanglement (or indicates the inefficiency of the witnesses). 

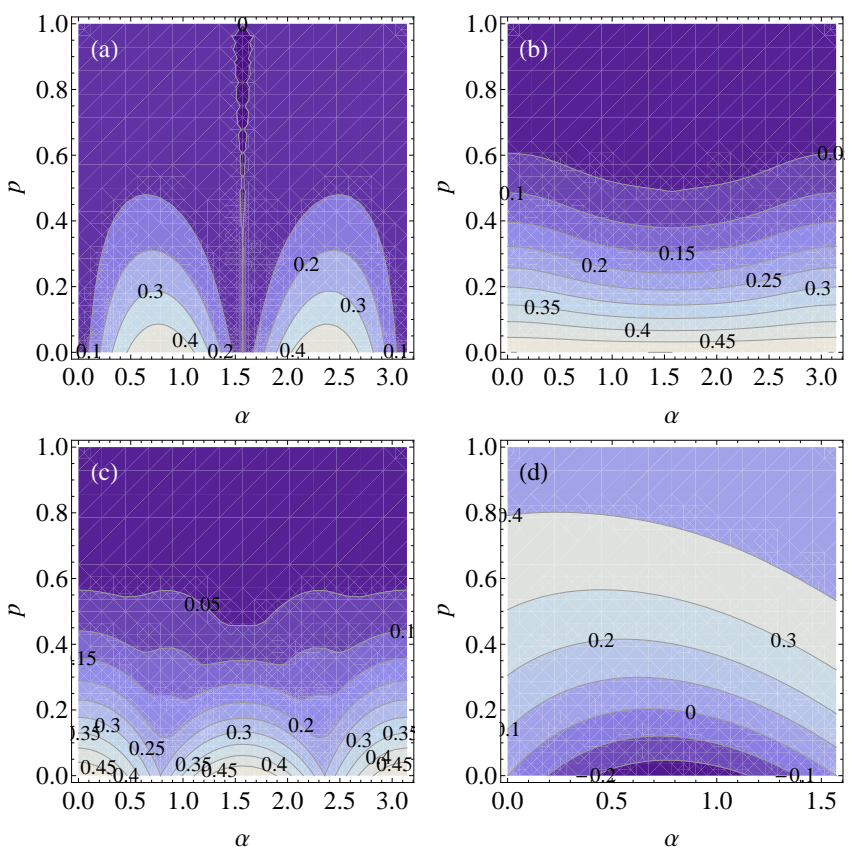

FIG. 2: (Color online) Evolution of various negativity measures as a function of initial state parameterized by $\alpha$ and amplitude damping strength, $p$. (a) $N^{(1)}$, the entanglement goes to zero at $\alpha=\pi / 2$. (b) $N^{(1,2)}$, (c) $N^{(1,3)}$, for these measures the entanglement goes to zero only in the limit of $p \rightarrow 1$. (d) Expectation value of four qubit cluster state with respect to entanglement witness $\mathcal{W}_{\beta}$, with $\beta=0$, as a function of initial state and decoherence strength. The four qubit cluster entanglement goes undetected at very low decoherence strengths $(p<.2)$ despite the presence of some sort of entanglement for any non-zero $p$.

The maximum decoherence for which detection is still possible is about the same for the amplitude damping and depolarizing and much higher for dephasing.

When comparing the entanglement evolution to the evolution of the gate fidelity or cluster state fidelity we find only superficial correlations. In addition, we do not find any signature of ESD in the fidelity functions. While clearly both entanglement and fidelity decrease as the decoherence strength increases, these superficial correlations do not give rise to any problems regarding the viability of quantum computing. We note, however, that this does not prove that ESD is completely irrelevant with respect to quantum computation in general, it simply demonstrates that the effect of ESD on this specific protocol is not manifest in the fidelity measure.

The gate fidelity provides a state independent measure for the accuracy of the entire single qubit rotation algorithm. This fidelity, and the explicit superoperators given in Appendix A, are vital information for those attempting to discern the possible accuracy that can be achieved by invariably decoherent experimental systems. We immediately note that the gate fidelity is the same in the dephasing and amplitude damping environments, $F_{A}^{g}=F_{z}^{g}$. This is not surprising considering that the Kraus oper-
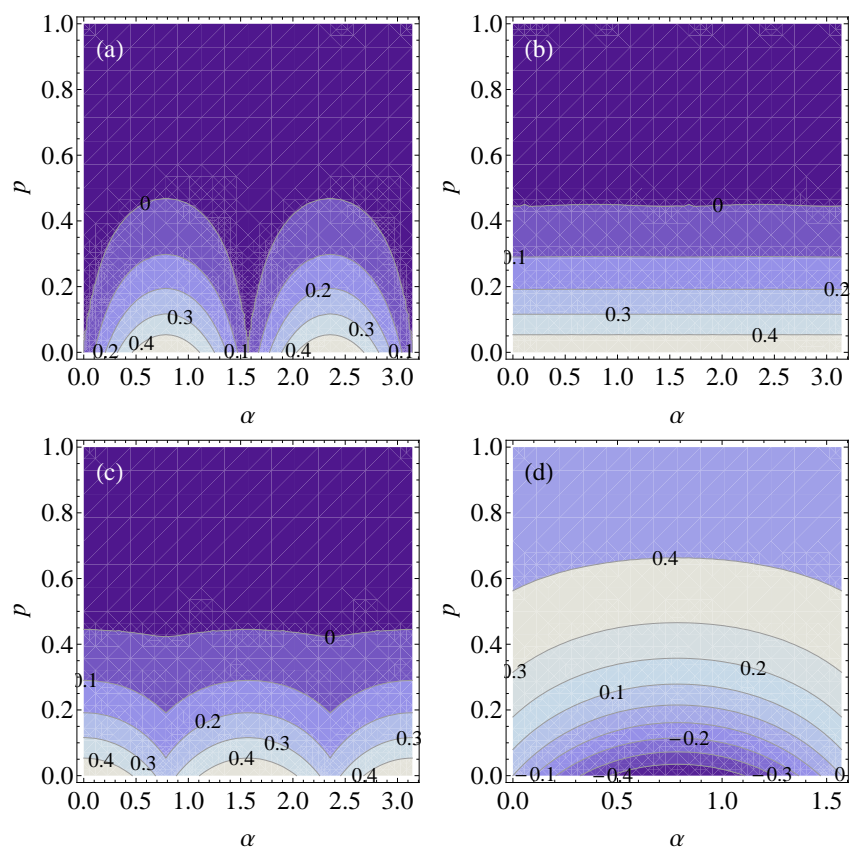

FIG. 3: (Color online) Negativity as a function of depolarizing strength, $p$, and initial state parameterized by $\alpha$ : (a) $N^{(1)}$, (b) $N^{(1,2)}$, (c) $N^{(1,3)}$. (d) Evolution of expectation value of entanglement witness as a function of initial state and decoherence strength. Notice that the evolution is similar to $N^{(1)}$ though the expectation value goes to zero well before ESD of $N^{(1)}$ The four qubit cluster entanglement can only be observed at low levels of decoherence, $p \lesssim .2$.

ators governing these decohering environments are very similar. Nevertheless, the cluster state fidelity of the two environments are not at all similar as seen in Figs. 4 and 5. This demonstrates the importance of utilizing multiple accuracy measures. In addition, the amplitude damping environment does not cause ESD for any entanglement measure while dephasing does. This suggests that dephasing is more harmful to the entanglement types found in the four qubit cluster state than is amplitude damping. Rotating the physical system qubits, such that a dephasing environment acts as an amplitude damping environment, could conserve entanglement though it would not increase the accuracy of the implemented single logical qubit rotation. Both fidelity types decrease much more quickly in the depolarizing environment than in the other two environments.

\section{CONCLUSION}

In conclusion, we have studied an attempted implementation of an arbitrary single qubit rotation via cluster state quantum computation in a noisy environment. We specifically looked at three different decohering environments: dephasing, amplitude damping, and depolarization. Such studies are vital for future experiments 

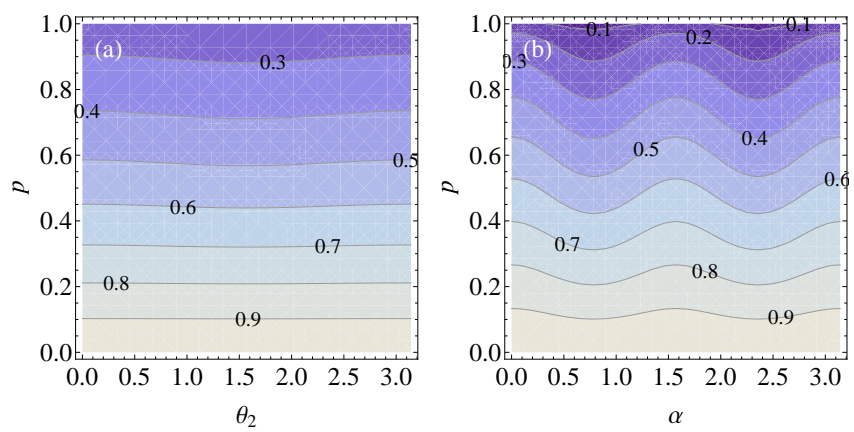

FIG. 4: (Color online) (a) Gate fidelity of an arbitrary single qubit rotation as a function of the rotation (parameterized by Euler angle $\theta_{2}$ with $\theta_{3}$ set to zero; note that the gate fidelity is independent of $\theta_{1}$ ), and dephasing strength $p$. (b) Fidelity of pre-measurement four qubit cluster state as a function of initial state parameterized by $\alpha$ (this fidelity is independent of $\beta$ ) and $p$.
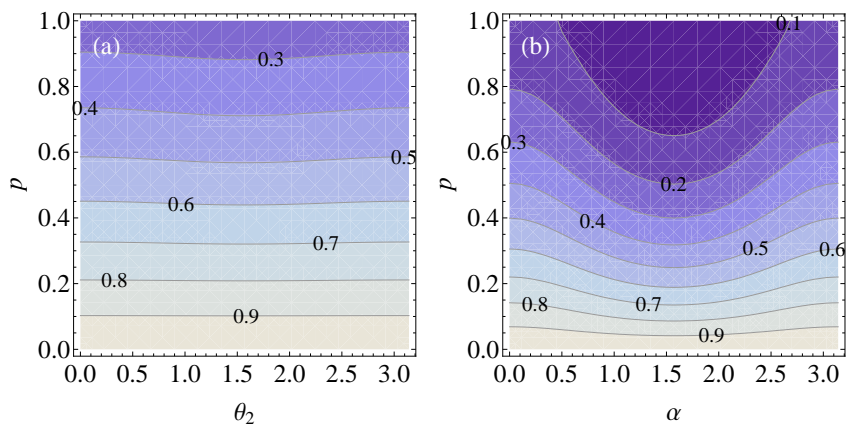

FIG. 5: (Color online) (a) Gate fidelity as a function of amplitude damping strength, $p$ and choice of rotation (parameterized by $\theta_{2}$ with $\theta_{3}=0$; note that the gate fidelity is independent of $\theta_{1}$ ). (b) Contour plot of four-qubit state fidelity as a function of amplitude damping strength and initial state (parameterized by $\alpha$; the fidelity is independent of $\beta$ ).
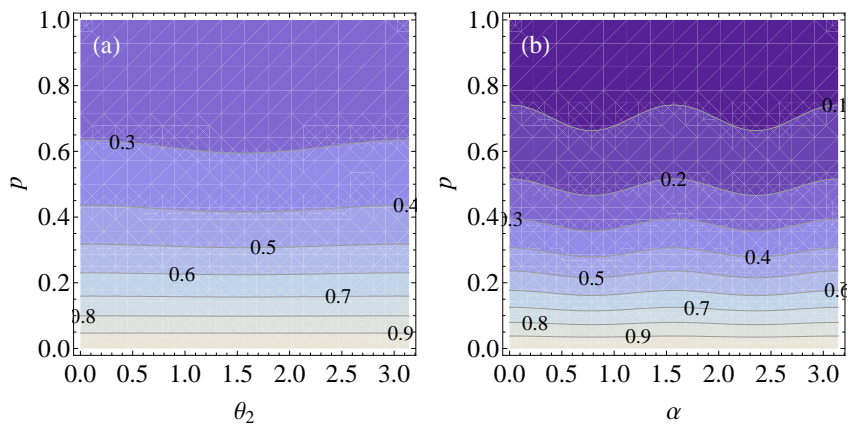

FIG. 6: (Color online) (a) Gate fidelity for arbitrary single qubit rotation in depolarization environment as a function of depolarization strength and the rotation (parameterized by $\theta_{2}$, in this case the gate fidelity does not depend either on $\theta_{1}$ or $\theta_{3}$ ). (b) Fidelity of four qubit cluster state as a function of initial state (parameterized by $\alpha$ as $F_{P}^{C}$ does not depend on $\beta$ ) and depolarizing strength. in cluster state quantum computation as they will shed light on the types of errors that occur during implementation and can prescribe what types and strengths of error are tolerable if attempting to achieve a certain accuracy of implementation. To this end, we have provided both accuracy measures of the implementation as a function of the attempted rotation and the decoherence type and strength, as well as the complete superoperator describing the entire process. The superoperators specifically allow for the identification of the type of error that will be manifest in the output state of an experiment given the error models. The accuracy measures and the superoperators are also vital for the determination of cluster based fault tolerance thresholds.

In addition, we have studied the entanglement evolution of the four qubit cluster state under the above mentioned decohering environments. This behavior is important for a number of reasons. First, multi-partite entanglement evolution under decoherence is still very much an area of intense study. Here we explore a number of negativity measures (which are bi-partite measures) and the detection capabilites of the entanglement witness. The latter is a way to test for the presence of cluster-type entanglement in experiments which will be necessary before proceeding with any cluster based algorithm. Our results allow for comparison of entanglement evolution between the different decohering environments. The entanglement evolution is compared to the fidelity measures in an attempt to note any possible correspondence between the two. The presence or lack of a correlation between fidelity and entanglement addresses the general question of the role of entanglement in quantum computation. Is entanglement integral to any quantum computation, or is it simply a byproduct of large Hilbert spaces? One may surmise that the role of entanglement is especially vital is cluster state quantum computation as the highly entangled cluster state is the basic resource for any algorithm. However, we showed that there is only superficial correlation between fidelity and entanglement and specifically noted that the complete disappearance of entanglement upon sufficient decoherence, entanglement sudden death, does not have any effect of the fidelity behavior. This is especially manifest when comparing the evolution under the dephasing and amplitude damping environments. While the gate fidelity of the single qubit arbitrary rotation is the same for both of these environments, the entanglement evolution is very different. All of this demonstrates that while entanglement is certainly necessary for universal quantum computation to be implemented on a cluster state the amount of entanglement per se is not a good indicator as to how accurately the algorithm will be implemented.

\section{Acknowledgments}

We acknowledge support from the MITRE Technology Program under MIP grant \#20MSR053. 


\section{Appendix A: Arbitrary Single Qubit Rotation Superoperators}

In this Appendix we provide the expressions for superoperators describing the evolution of a single logical qubit in a cluster based quantum computation attempting to implement an arbitrary rotation described by Euler angles $\left(\theta_{1}, \theta_{2}, \theta_{3}\right)$ in the different decohering environments. The superoperator for the case of qubits in a dephasing environment is given by:

$$
S_{z}=\frac{1}{2}\left(\begin{array}{cccc}
(1-q s 2 s 3) & -e^{i \theta_{1}} q(c 3-i \tilde{p} c 2 s 3) & -e^{-i \theta_{1}} q(c 3+i \tilde{p} c 2 s 3) & (1+q s 2 s 3) \\
q(c 2-i \tilde{p} c 3 s 2) & e^{i \theta_{1}} q(q c 2 c 3+i \tilde{p}(s 2+s 3)) & -e^{-i \theta_{1}} q(q c 2 c 3+i \tilde{p}(s 2-s 3)) & -q(c 2-i \tilde{p} c 3 s 2) \\
q(c 2+i \tilde{p} c 3 s 2) & -e^{i \theta_{1}} q(q c 2 c 3-i \tilde{p}(s 2-s 3)) & e^{-i \theta_{1}} q(q c 2 c 3-i \tilde{p}(s 2+s 3)) & -q(c 2+i \tilde{p} c 3 s 2) \\
(1+q s 2 s 3) & e^{i \theta_{1}} q(c 3-i \tilde{p} c 2 s 3) & e^{-i \theta_{1}} q(c 3+i \tilde{p} c 2 s 3) & (1-q s 2 s 3)
\end{array}\right),
$$

where $q \equiv p-1, \tilde{p} \equiv \sqrt{1-p}$, and we write $\sin \theta_{j}$ and $\cos \theta_{j}$ for $j=1,2,3$ as $s j$ and $c j$.

The superoperator for the amplitude damping environment is given by

$$
S_{A}=\frac{1}{2}\left(\begin{array}{cccc}
(1+p)+q^{2} s 2 s 3 & e^{i \theta_{1}} q^{2}(c 3-i \tilde{p} c 2 s 3) & e^{-i \theta_{1}} q^{2}(c 3+i \tilde{p} c 2 s 3) & (1+p)-q^{2} s 2 s 3 \\
q(c 2-i \tilde{p} c 3 s 2) & e^{i \theta_{1}} q(q c 2 c 3+i \tilde{p}(s 2+s 3)) & -e^{-i \theta_{1}} q(q c 2 c 3+i \tilde{p}(s 2-s 3)) & -q(c 2-i \tilde{p} c 3 s 2) \\
q(c 2+i \tilde{p} c 3 s 2) & -e^{i \theta_{1}} q(q c 2 c 3-i \tilde{p}(s 2-s 3)) & e^{-i \theta_{1}} q(q c 2 c 3-i \tilde{p}(s 2+s 3)) & -q(c 2+i \tilde{p} c 3 s 2) \\
-q(1+q s 2 s 3) & -e^{i \theta_{1}} q^{2}(c 3-i \tilde{p} c 2 s 3) & -e^{-i \theta_{1}} q^{2}(c 3+i \tilde{p} c 2 s 3) & -q(1-q s 2 s 3)
\end{array}\right) .
$$

We note that the second and third rows of the amplitude damping superoperator are exactly the same as the second and third rows of the dephasing superoperator.

The superoperator for the depolarizing environment is given by

$$
S_{P}=\frac{1}{2}\left(\begin{array}{cccc}
1-q^{3} s 2 s 3 & -e^{-i \theta_{1}} q^{3}(c 3+i q c 2 s 3) & e^{-i \theta_{1}} q^{3}(-c 3+i q c 2 s 3) & 1+q^{3} s 2 s 3 \\
-q^{2}(c 2+i q c 3 s 2) & e^{i \theta_{1}} q^{3}(q c 2 c 3+i(s 2+s 3)) & -e^{-i \theta_{1}} q^{3}(q c 2 c 3+i(s 2-s 3)) & q^{2}(c 2+i q c 3 s 2) \\
-q^{2}(c 2-i q c 3 s 2) & -e^{i \theta_{1}} q^{3}(q c 2 c 3-i(s 2-s 3)) & e^{-i \theta_{1}} q^{3}(q c 2 c 3-i(s 2+s 3)) & q^{2}(c 2-i q c 3 s 2) \\
1+q^{3} s 2 s 3 & e^{-i \theta_{1}} q^{3}(c 3+i q c 2 s 3) & e^{-i \theta_{1}} q^{3}(c 3-i q c 2 s 3) & 1-q^{3} s 2 s 3
\end{array}\right) .
$$

The above superoperators promise to be useful for experimental realizations of this cluster state protocol, including questions of fault tolerance, as they can be used to characterize a given environment.

\section{Appendix B: Kraus Operator Representation}

In the main part of this paper we determined the superoperators for single logical qubit rotations in a cluster based quantum computer undergoing different types of decoherence. Recasting these superoperators in terms of Kraus operators gives additional insight into the evolution of the logical information under the arbitrary qubit rotation as a function of decoherence. To calculate the Kraus operators from the superoperator one first determines the Choi matrix. Each Kraus operator $K_{a}$ is a Choi matrix eigenvector (unstacked so that its dimension is $N \times N)$, times the square root of the corresponding Choi matrix eigenvalue divided by $N$ 23. We define the amplitude of a given Kraus operator, $A_{a}$, to be the square root of the Choi matrix eigenvalue divided by $N$, $A_{a}=\sqrt{\lambda_{a} / N}$. The higher the amplitude of a Kraus operator the more significant its effect on the overall system dynamics. This method of Kraus operator construction maximizes the amplitude of one (and hence the most significant) Kraus operator. Using Kraus operators, the complete evolution of the system is given by

$$
\sum_{a} K_{a}\left(p, \theta_{1}, \theta_{2}, \theta_{3}\right) \rho_{\text {in }}(\alpha, \beta) K_{a}\left(p, \theta_{1}, \theta_{2}, \theta_{3}\right)^{\dagger}=\rho_{\text {out }} .
$$

Clearly, if there is only one Kraus operator it will be unitary with an amplitude of 1 . In this way, unitarity of the evolution can be quantified by $A_{1}$, the amplitude of the first Kraus oprerator. In addition, the accuracy of the applied evolution can be quantified by the fidelity or correlation of the first Kraus operator as compared to the desired unitary 22]. The fidelity is given by:

$$
F^{1}=\frac{\operatorname{Tr}\left[U^{\dagger} K_{1}\right]}{\operatorname{Tr}\left[U^{\dagger} U\right]}
$$

and the correlation is given by:

$$
C^{1}=\frac{\operatorname{Tr}\left[U^{\dagger} K_{1}\right]}{\sqrt{\operatorname{Tr}\left[U^{\dagger} U\right] \operatorname{Tr}\left[K_{1}^{\dagger} K_{1}\right]}} .
$$

The fidelity measure accounts for both decoherent losses, a change in purity, and coherent errors, what we might call a change in 'direction' effected by the first Kraus 


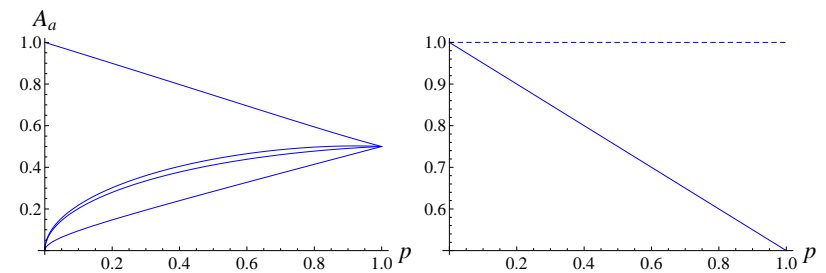

FIG. 7: (Color online) (left) Kraus operator amplitudes as a function of dephasing strength. The highest Kraus operator amplitude decreases linearly until becoming equal to the amplitudes of the other three Kraus operators. The behavior of the highest and lowest amplitudes are independent of all measurement angles while the middle two amplitudes depend slightly on $\theta_{2}$ and $\theta_{3}$. (right) Fidelity (solid line) and correlation (dashed line) of first Kraus operator as a function of dephasing strength.
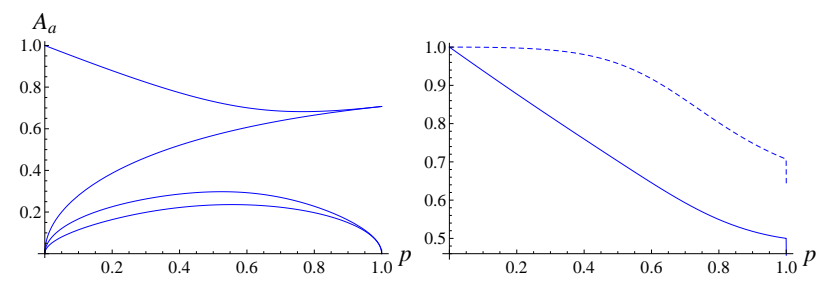

FIG. 8: (Color online) (left) Kraus operator amplitudes as a function of amplitude damping strength. The behavior of all amplitudes depend slightly on $\theta_{2}$ and $\theta_{3}$. (right) Fidelity (solid line) and correlation (dashed line) of first Kraus operator as a function of amplitude damping strength. Note that in this case the correlation does not remain constant implying a coherent affect on the dynamics of the system due to amplitude damping.

operator. The correlation is unaffected by a change in magnitude.

We start with the Kraus operators of the dephasing environment. The amplitude of the Kraus operators as a function of decoherence strength is shown in Fig. 17. The amplitude of the first Kraus operator decreases linearly and the amplitude of the other three Kraus operators increase until, at $p=1$, the four Kraus operators have equal amplitudes. At that limit each of the four Kraus operator matrices have an element equal to $1 / \sqrt{2}$ in one of the corners and all the other elements are zero. The fidelity of the first Kraus operator also decreases linearly with dephasing strength while the correlation remains constant at 1 (until very high $p$ ) implying purely decoherent evolution.

We noted in the main part of the paper that the gate fidelities of a single logical qubit cluster state-based arbitrary rotation in a dephasing environment and amplitude damping environment are the same. Nevertheless, we find that their Kraus operator representations are very different. Two of the Kraus operator amplitudes in an amplitude damping environment go to zero as $p \rightarrow 1$. The remaining two Kraus operator matrices have a one in the upper right or left corner and zeros elsewhere. For
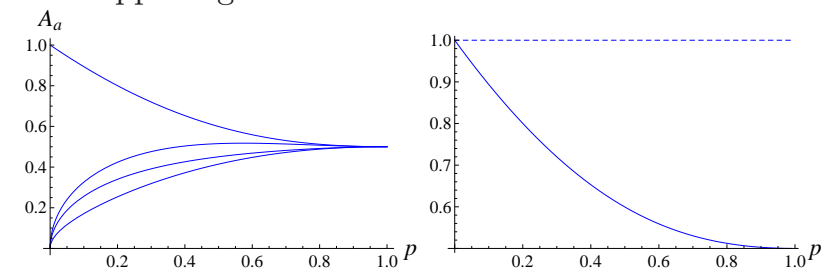

FIG. 9: (Color online) (left) Kraus operator amplitudes as a function of depolarizing strength. The behavior of all amplitudes depend slightly on $\theta_{2}$. (right) Fidelity (solid line) and correlation (dashed line) of first Kraus operator as a function of dephasing strength.

values of $p<1$, the amplitude of the first Kraus operator decreases faster in the amplitude damping environment than in the dephasing environment. However, this descent slows as $p$ approaches one. The second Kraus operator always plays a more significant role in the amplitude damping environment than in the dephasing environment.

The fidelity of the first Kraus operator as function of amplitude damping strength $p$ decreases linearly (faster than the dephasing environment) before rounding off at high values of $p$ while the correlation decreases, staying near one only at low values of $p$. This behavior, portrayed in Fig. 8, suggests that amplitude damping, despite being decoherent dynamics, has a coherent affect on the system dynamics. Rotating the system so that the amplitude damping acts as phase damping may increase the fidelity and correlation of the first Kraus operator but will not increase the gate fidelity of the attempted logical qubit rotation.

Of all the decohering environments studied here, the first Kraus operator amplitude decreases fastest (and not linearly) in a depolarizing environment. The increase of the lowest amplitude Kraus operator is also not linear. However, in the limit of $p \rightarrow 1$, the depolarizing environment is like the dephasing environment in that the amplitudes of all four Kraus operators converge to .5, as demonstrated in Fig. 9. The fidelity in a depolarzing environment also decreases faster than the other decoherent environments while the correlation remains constant at one, demonstrating that the evolution is entirely decoherent.
[1] For a recent review see R. Horodecki, P. Horodecki, M. Horodecki, K. Horodecki, Rev. Mod Phys. 81, 865
(2009).

[2] R. Raussendorf and H. J. Briegel, Phys. Rev. Lett. 86, 
5188 (2001).

[3] M Nielsen, I. Chuang, Quantum information and Computation (Cambridge University Press, Cambridge, 2000).

[4] C. Simon and J. Kempe, Phys. Rev. A 65, 052327 (2002); W. Dur and H.-J. Briegel, Phys. Rev. Lett. 92180403 (2004); S. Bandyopadhyay and D.A. Lidar, Phys. Rev. A 72, 042339 (2005).

[5] M. Hein, W. Dur, and H.-J. Briegel, Phys. Rev. A 71, 032350 (2005); O. Guhne, F. Bodoky, and M. Blaauboer, Phys. Rev. A 78, 060301(R) (2008); D. Cavalcanti, R. Chaves, L. Aolita, L. Davidovich, and A. Acin, Phys. Rev. Lett 103, 030502 (2009); L. Aolita, D. Cavalcanti, R. Chaves, C. Dhara, L. Davidovich, and A. Acin, Phys. Rev. A 82, 032317 (2010).

[6] L. Diosi, in Irreversible Quantum Dynamics, edited by F. Benatti and R. Floreanini, Lect. Notes Phys. 622, (Springer-Verlag, Berlin) 157 (2003); P.J. Dodd and J.J. Halliwell, Phys. Rev. A 69, 052105 (2004).

[7] T. Yu and J.H. Eberly, Phys. Rev. Lett. 93, 140404 (2004); ibid. 97, 140403 (2006).

[8] I. Sainz and G. Bjork, Phys. Rev. A 76, 042313 (2007).

[9] L. Aolita, R. Chaves, D. Cavalcanti, A. Acin, and L. Davidovich, Phys. Rev. Lett. 100, 080501 (2008).

[10] C.E. Lopez, G. Romero, F. Lastra, E. Solano, and J.C. Retamal, Phys. Rev. Lett. 101, 080503 (2008).

[11] M. Yonac, T. Yu, J.H. Eberly, J. Phys. B 39, 5621 (2006); ibid. 40, 545 (2007).

[12] I. Sainz and G. Bjork, Phys. Rev A 77, 052307 (2008).

[13] Y.S. Weinstein, Phys. Rev A 79, 012318 (2009); Y.S. Weinstein, Phys. Rev A 82, 032326 (2010).

[14] Y.S. Weinstein, Phys. Rev A 79, 052325 (2009).

[15] M.P. Almeida, et al., Science 316, 579 (2007); J. Laurat, K.S. Choi, H. Deng, C.W. Chou, and H.J. Kimble, Phys. Rev. Lett. 99, 180504 (2007); A. Salles, F. de Melo, M.P. Almeida, M. Hor-Meyll, S.P. Walborn, P.H. Souto Ribeiro, and L. Davidovich, Phys. Rev. A 78, 022322 (2008).

[16] Y.S. Weinstein, Phys. Rev A 80, 022310 (2009).

[17] H. J. Briegel and R. Raussendorf, Phys. Rev. Lett. 86, 910 (2001).

[18] R. Raussendorf, D. E. Browne, and H. J. Briegel, Phys. Rev. A 68, 022312 (2003).

[19] G. Vidal and R.F. Werner, Phys. Rev. A 65032314 (2002).

[20] B.M. Terhal, Phys. Lett. A 271, 319 (2000); M. Lewenstein, B. Kraus, J.I. Cirac, and P. Horodecki, Phys. Rev. A 62, 052310 (2000).

[21] G. Toth and O. Guhne, Phys. Rev. Lett. 94, 060501 (2005).

[22] Y.S. Weinstein, et. al., J. Chem. Phys. 121, 6117 (2004).

[23] T.F. Havel, J. Math. Phys. 44, 534 (2003). 